Sādhanā Vol. 36, Part 2, April 2011, pp. 251-265. (C) Indian Academy of Sciences

\title{
Melt pool vorticity in deep penetration laser material welding
}

\author{
N KUMAR*, S DASH, A K TYAGI and BALDEV RAJ \\ Surface and Nanoscience Division, Indira Gandhi Centre for Atomic Research, \\ Kalpakkam 603 102, Tamil Nadu, India \\ e-mail: niranjan@igcar.gov.in
}

MS received 8 June 2010; revised 12 November 2010; accepted 11 December 2010

\begin{abstract}
In the present study, the vorticity of melt motion in the keyhole and weld pool has been evaluated in case of high power $\mathrm{CO}_{2}$ laser beam welding. The circulation of vorticity is obtained as a function of Reynolds number for a given keyhole volume which is linked to Mach number variation. The shear stress and thermal fluxes present in the turbulent pool are linked to diffusivity and Prandtl number variation. It was shown that below a critical value of Rayleigh number, the conduction mode of melt transfer signifying beam absorption becomes dominant. Above this value, convective heat transfer indicates melting and evaporation occurring in the weld pool during laser welding. The evaporative recoil pressure expels the liquid while surface tension and hydrostatic pressure help to retain the melt in the keyhole cavity in this high power laser beam welding. The understanding of several hydrodynamic phenomena occuring in the weld pool is valuable not only for understanding basic mechanistic aspects but also for process optimization involved in laser beam welding.
\end{abstract}

Keywords. Keyhole; melt pool; vorticity; dimensionless numbers; liquid-vapour phase.

\section{Introduction}

A keyhole laser spot welding often leads to appearance of porous defects in the weld seam. A fundamental understanding behind evolution of such defects is essential to devise optimum conditions for their ellimination. A simplified situation, offering several advantages, has been found in case of laser melting of Zinc. High speed X-ray transmission imaging of an enlarged keyhole has indicated violent melt motion. This observation has facilitated the development of a

*For correspondence 
semi-analytical mathematical model described by Kaplan et al (2002). The formation and stability of stationary laser weld keyholes have been investigated using numerical simulation. The effect of multiple reflections in the keyhole was estimated by Mi \& Charles (2004) by using a ray tracing method. The free surface profile, flow velocity and temperature distribution were calculated numerically by $\mathrm{He}$ et al (2003). In laser and electron-beam welding, a deep cavity called a keyhole or beam hole is formed in the weld pool due to the intense recoil pressure exetrted by rapid evaporation. The formation of the keyhole leads to a deep penetration weld with a high aspect ratio and this is the most advantageous feature of welding by high-energydensity beams. Systematic studies on observation of keyhole as well as the weld pool dynamics prevailing there and the related phenomena that reveal the mechanism of porosity formation have enabled formation of suppression methods Dowden (2002). Marangoni convection and viscous drag associated with vapour motion in the keyhole was identified as the two most probable causes of turbulence in the weld pool described by Fabbro et al (2004). Axial fluid motion inside the keyhole was explained by drawing mathematical linkages between viscous drag and Marangoni convection in a turbulent flow field. Several bubbles were formed across the keyhole by intense material evaporation process. As studied by Hu \& Tsai (2003) it has also been revealed that the geometry of the keyhole fluctuates frequently with respect to changes in size and shape.

Numerical and experimental studies were conducted to investigate the heat transfer, fluid flow and keyhole dynamics during pulsed laser welding. For this purpose, Kaplan (1994) has developed a comprehensive mathematical model. In this model, the continuum formulation was used to handle solid phase, liquid phase and mushy zone encountered during melting and solidification processes. The experiments based on twin or triple spot interaction geometry have also indicated that the friction forces exercised by the metal vapour escaping from the keyhole have an important role in the pool hydrodynamics worked out by Kaplan et al (2002). The objective of several mathematical models and the associated numerical techniques was aimed to calculate transient heat transfer and fluid flow in the melt pool. These studies profoundly influenced the understanding of mechanisms leading to the oval shape of the melt pool during dual beam laser welding. This was observed by Lee et al (2002). The deformation of the melt pool was ascribed to non-uniform evaporation on the front wall of the keyhole. Both the keyhole and the weld pool were strongly disturbed by the dynamic pressure of the metallic vapour jet. The characteristic spherical and elongated pores were found to have a composition dominated by the metal vapour entrained with shielding gas studied by Matsunawa (2001). The equations governing the process of fluid flow has two distinct phase boundaries; one is between the keyhole and the molten metal while the other one is between the liquid and the solid states of the metal. High-power lasers evolved keyhole in a solid target is unstable with respect to collapse and can be maintained only by achieving a balance between pressure terms ensuing from laser vaporization, surface tension and hydrostatic pressure. It was understood from the previous works that the vapour recoil pressure is the main driving force for deep penetration keyhole formation studied by Zhou et al (2006). However, the hydrodynamics of melt motion in keyhole and weld pool have not been completely addressed.

A comprehensive plot of turbulent flow in the keyhole during laser beam welding is necessary to understand the underlying physical process. This will lead to better quality welding. Hydrodynamical analysis of melt motion in keyhole and weld pool is the subject matter of present study. Such analysis is essential for rectification of laser welding process parameters. In this regard, the momentum and turbulent dynamics concepts have been analytically applied to address the melt pool vorticity, which is responsible for dimensional instabilities of the keyhole during high power laser beam welding. 


\section{Melt flow in weld pool}

A keyhole in the shape of a conical cavity is formed due to high power (up to $P=2.5 \mathrm{~kW}$ ) continuous wave linearly polarized $\mathrm{CO}_{2}$ laser radiation impinging on the mild steel. The ray tracing method is used to estimate the beam propagation into the material. The incident laser beam is assumed to consist of rays and the power of the ray is computed from a circularly symmetric Gaussian beam profile. The ray tracing procedure has following assumption. The molten metal surfaces are specularly reflecting. The plume absorption into the material is negligible. The polarization direction in the beam is random. The laser beam with an intensity $I=10^{7} \mathrm{Wcm}^{-1}$ forms the keyhole channel in the material with a depth extending to $1.2 \mathrm{~mm}$. In table 1, the material parameters of mild steel are mentioned. These parameters were used in our calculations.

Klein \& Vicanek (1996) calculated the axi-symmetric keyhole profile and flow velocity numerically using the Volume of Fluid (VOF) method. The governing equations for the VOF method consist of continuity, momentum and volume of fraction relations. These governing equations are listed below

$$
\begin{gathered}
\nabla \cdot \vec{v}=0, \\
\frac{\partial \vec{v}}{\partial t}+(\vec{v} \cdot \nabla) \vec{v}=-\frac{1}{\rho} \nabla p+v \nabla^{2} \vec{v}+\beta \vec{g} \Delta T, \\
\frac{\partial F}{\partial t}+(\vec{v} \cdot \nabla) F=0,
\end{gathered}
$$

where $\vec{v}$ represents the velocity vector, $p$ is the pressure, $\rho$ is the mass density, $v$ is the kinematic viscosity, $\beta$ is the thermal expansion coefficient, $g$ is the acceleration due to gravity and $F$ is the volume fraction of the fluid in the grid cell used for calculations. The above volume fraction equation allows computation of the position of a moving liquid surface within a fixed grid system so that the keyhole shape can be obtained after each time step.

In figure 1, it is assumed that the free boundary curve is moving with the front and rear wall of the keyhole along the axis of laser beam. Boundary of the weld pool is assumed to be the region of free melt motion prevailing between the rear wall of keyhole and solid front. The movement of the weld pool is proportional to the velocity, $V$ of the specimen. In the numerical approach, the keyhole is assumed to be cylindrical with a uniform temperature along its keyhole wall. This temperature is set at a value slightly above the evaporation temperature of the material. The laser power is supposed to be completely absorbed by the wall of the cylindrical keyhole. The depth of the keyhole is then determined iteratively in such a way that the integral of the heat

Table 1. Material properties of mild steel and constants used in calculation (Fabbro et al 2004).

\begin{tabular}{lll}
\hline No. & Physical parameters & Typical values \\
\hline 1 & Mass density, $\rho$ & $7860 \mathrm{~kg} \mathrm{~m}^{-3}$ \\
2 & Kinematic viscosity, $\mu$ & $5.6 \times 10^{-7} \mathrm{~m}^{2} \mathrm{~s}^{-1}$ \\
3 & Surface tension coefficient, $\sigma$ & $1.2 \mathrm{Nm}^{-1}$ \\
4 & Surface tension gradient, $\frac{d \sigma}{d T}$ & $0.2 \mathrm{dyne} \mathrm{cm}^{-1} \mathrm{~K}^{-1}$ \\
5 & Thermal conductivity, $k$ & $30 \mathrm{~W} \mathrm{~m} \mathrm{~K}^{-1}$ \\
6 & Specific heat, $\boldsymbol{C}_{p}$ & $795 \mathrm{~J} \mathrm{~kg}^{-1} \mathrm{~K}^{-1}$ \\
7 & Latent heat of vapourization, $h_{l}$ & $6084 \mathrm{~kJ} \mathrm{~kg}^{-1}$ \\
8 & Evaporating temperature, $T_{e v}$ & $3130 \mathrm{~K}$ \\
\hline
\end{tabular}




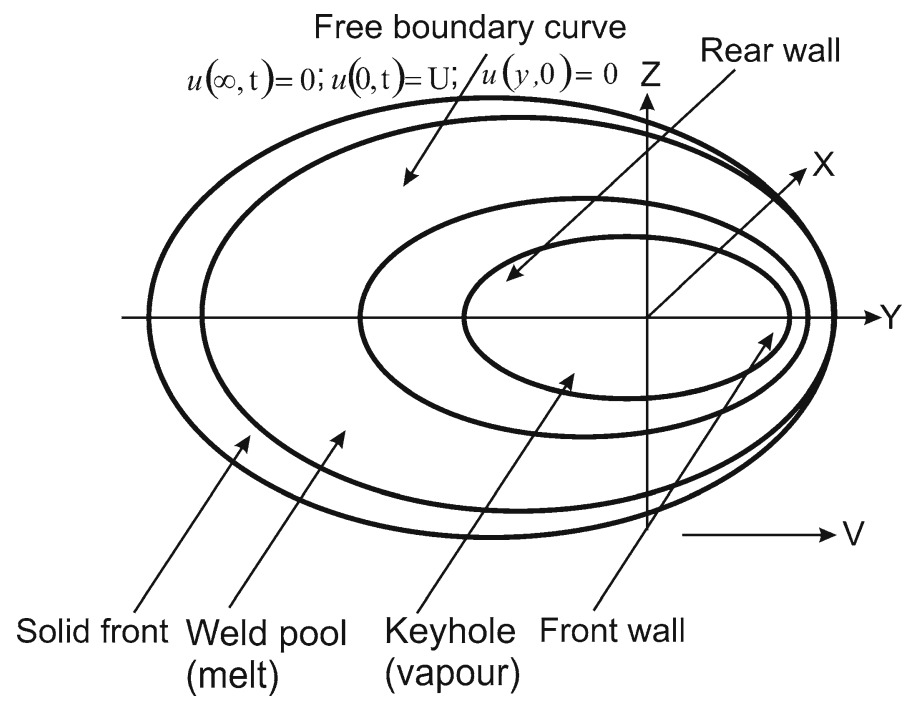

Figure 1. Sketch of the keyhole model.

flux along the entire surface of the keyhole equals the total absorbed laser power. An analytical description can also be used for this purpose where material properties remain constant. The diameter of the keyhole is supposed to be equal to the diameter of the laser spot. The initial temperature $\mathrm{T}_{0}$ is equal to $300 \mathrm{~K}$. The material properties are temperature dependant and the latent heat of fusion is also taken into account. The material is irradiated by the laser beam perpendicular to the surface and moving at a velocity $V_{0}$ along $\mathrm{x}$-axis. The laser beam-welding problem is assumed to be at a quasi-steady state. In this model, the hydrodynamics phenomena are also taken into account which is described by Kamel et al (2008). The energy equation is solved using a coordinate system moving with the heat source. The liquid metal is considered as an incompressible Newtonian fluid. The equations of mass and momentum conservation are supposed to govern the fluid flow equations (1-3). According to hydrodynamic approach, the speed of sound increases in the keyhole as the temperature increases, the actual speed of melt moving at Mach value depends on the melt temperature around it. As the Mach number increases, so does the strength of the shock wave and the Mach angle becomes increasingly narrow. As the melt flow crosses the shock wave, its speed is reduced and the associated temperature, pressure and density increase. The stronger the shock, the greater the changes. At high enough Mach numbers, the temperature increases so much over the shock that ionization of gas molecules behind the shock wave commences.

The surface tension equation $\sigma=\frac{\sigma_{c}}{r_{k h}}$ is based on a pressure balance, where $\sigma_{c}$ refers to surface tension coefficient, $r_{k h}$ is the radius of the keyhole. The term $r_{k h}$ need to balance the sum of vapour recoil pressure $p_{a b}$ of particles ablated at the keyhole surface and the excess pressure $\delta p_{g}$ due to the gas streaming out of the keyhole. Pressure at the surface is the sum total of vapour recoil pressure and ablation pressure. Neglecting plasma effects, the ablation pressure is determined by the solution of the kinetic knudsen layer equations as stated in $p_{a b}=m n_{g} u_{g}^{2} . m$ is the mass of a metal atom. The density $n_{g}$ and the velocity $u_{g}$ of the particles at the end of the knudsen layer may be expressed in terms of the keyhole surface temperature $T_{s}$ and the average gas pressure $p_{o}+\delta p_{g}$ within the keyhole ( $p_{o}$ is the atmospheric pressure). Taking into account 
the kinematic viscosity $\mu_{g}$ of the vapour, we may express the average excess pressure $\delta p_{g}$ within the keyhole as (Kaplan 1994)

$$
\delta p_{g}=\frac{C_{g}(R e)}{3}\left(\frac{d}{a}\right)^{2} \cdot p_{a b},
$$

$C_{g}$ is a gas flow parameter, $C_{g}(R e)$ in equation (4) depends on the Reynolds number $R e=\frac{r_{k h} v_{l}}{v_{g}}$ for the gas flow, $v l$ is the injection velocity of the ablated liquid particles at the keyhole surface, $v_{g}$ is kinematic viscosity of the gas. The constant rate of gas flow $C_{g}\left(R_{e}\right)$ can be calculated for solution of the Navier-Stokes equations within the keyhole.

$$
\frac{\partial u_{m}}{\partial t}+u_{m} \frac{\partial u_{m}}{\partial r}=-\frac{1}{\Re} \frac{\partial p}{\partial r}+v \frac{\partial}{\partial r}\left[\frac{1}{r} \frac{\partial}{\partial r}\left(r u_{m}\right)\right],
$$

$\Re$ is the average mass density of the melt, $p$ is the acting pressure in the keyhole, $u_{m}$ is the radial velocity in the melt flow.

$$
\frac{1}{2} \operatorname{In}\left(\frac{r_{m}}{r_{s}}\right) \frac{d^{2}}{d t^{2}}\left(r_{s}^{2}\right)+\frac{1}{8}\left(\frac{1}{r_{m}^{2}}-\frac{1}{r_{s}^{2}}\right)\left[\frac{d}{d t}\left(r_{s}^{2}\right)\right]^{2}=-\frac{1}{\Re}\left(p_{2}-p_{1}\right),
$$

$p_{2}-p_{1}$ denotes the pressure difference between $r=r_{s}$ and $r=r_{m}$ with in the melt phase that is related to the external pressure difference. The Partial Differential Equation (PDE) form of above NS equation can be integrated between actual radius of the weld pool $r_{m}$ and of the keyhole radius $r_{s}$ to yield an Ordinary Differential Equation (ODE) for the calculation of temporal growth of keyhole radius $r_{s}(t)$. The circulation of the molten phase of the metal in the keyhole $\Gamma$ can be defined as integral along a closed curve running over the keyhole as (Seto et al 2000; Matsunawa 2001)

$$
\Gamma=\int_{C} \vec{w} \cdot d \vec{r}
$$

where $d \vec{r}$ is the tangent to $C$ which points to the circulatory direction. The Stoke's theorem relates the circulation to the vorticity of the molten phase present inside the keyhole. We can estimate the momentum of the molten phase circulating across the tangential force at certain point inside the keyhole

$$
\Gamma=\int_{C} \vec{w} \cdot d \vec{r}=\int_{S} \hat{n} \cdot(\nabla x \cdot \vec{\omega}) d s=\int_{S} \hat{n} \cdot \vec{\omega} d s,
$$

where $S$ is an open surface of the keyhole mouth that caps $C, \nabla x$ is the length of fluid which represents length of melt phase along the laser beam axis. This relation can be differentiated to yield following equation

$$
d \Gamma=\hat{n} \cdot \vec{\omega} d s
$$

where $d \Gamma$ equals the component of angular velocity $\vec{\omega}$ that is normal to $d s$ as curve $C$ shrinks to a point. This relation represents the circulation and momentum of vorticity of the molten metal in the keyhole volume. To clarify the connection, consider a region of the liquid molten phase in the keyhole in which $\vec{\omega}=0$. Then at $d \Gamma=0$, the circulation of the fluid flow becomes constant. This need not be zero in the keyhole region. On the other hand, if the vorticity is 
non-zero then the magnitude of $d \Gamma$ at each point of the keyhole region will depend on the orientation of $\hat{n}$. The kinematic expression for vorticity evaluated by obtaining curl of the acceleration of the fluid flow is given as

$$
\left\{\begin{array}{l}
\nabla x \cdot \vec{a}=\nabla x \frac{d \vec{w}}{d t}=\nabla x\left(\frac{\partial \vec{w}}{\partial t}+\vec{\omega} x \cdot \vec{w}+\nabla \frac{w^{2}}{2}\right)=\frac{\partial}{\partial t}(\nabla x \cdot \vec{w})+\nabla x(\vec{\omega} x \cdot \vec{w})= \\
=\frac{\partial \vec{\omega}}{\partial t}+\nabla x(\vec{\omega} \cdot x \vec{w}) .
\end{array}\right.
$$

Replacement of fluid acceleration $\vec{a}$ in momentum equation will cause the results being not kinematic. We will consider Rayleigh flow if it is compressible. The kinematic viscosity $v$ remains non-zero. When the Rayleigh number is below a critical value for the fluid inside keyhole, heat transfer primarily takes place in the form of conduction. Above the critical value, heat transfer becomes convection dominated. The Rayleigh number is defined as the product of the Grashof number that describes the relationship between buoyancy and viscosity within a fluid and the Prandtl number describing the relationship between momentum and thermal diffusivities. Hence, the Rayleigh number itself may be viewed as the ratio of buoyancy forces and (the product of) thermal and momentum diffusivities. For convection inside keyhole, we can estimate the Rayleigh number as $R a=G r \cdot \operatorname{Pr}=\frac{g \beta}{v \cdot \alpha}\left(T_{k h}-T_{\infty k h}\right) V_{k h}^{2}$. The Grashof number $G r$ defines the heat transfer which approximates the ratio of the buoyancy to viscous forces acting on the keyhole. This is $G r=\frac{g \beta\left(T_{k h}-T_{\infty k h}\right) V_{k h}^{2}}{v^{2}}$, where $\operatorname{Pr}=\frac{v}{\alpha}$ is Prandtl number. $V_{k h}$ is the characteristic volume of the keyhole. $T_{k h}$ is the keyhole surface temperature. $T_{\infty k h}$ is quiescent of temperature (fluid temperature at the keyhole boundary surface), where $v$ is the kinematic viscosity, $\alpha$ is the thermal diffusivity, $\beta$ is the thermal expansion coefficient. However, for an incompressible flow, $v=0$ which renders momentum and energy equations to be reduced to following terms:

$$
\begin{gathered}
\frac{\partial u}{\partial t}=\frac{\partial}{\partial t}\left(\frac{v}{\rho} \frac{\partial u}{\partial t}\right), \\
\frac{\partial}{\partial t}\left(h-\frac{p}{\rho}\right)=\frac{\partial}{\partial y}\left(\frac{k}{\rho} \frac{\partial T}{\partial y}\right)+\frac{v}{\rho}\left(\frac{\partial u}{\partial y}\right)^{2},
\end{gathered}
$$

where $k$ is thermal conductivity and $h$ is depth of the keyhole. In the equations (11) and (12) all the variables are dimensional and the right hand side term in the energy equation (12) is the viscous dissipation function divided by liquid phase density $\rho$ at an elapsed time $t \geq 0, y=0$. It is possible to establish the complete set of initial and boundary conditions in the following manner

$$
\left\{\begin{array}{l}
u(y, 0)=0, \quad u(0, t)=U, \quad u(\infty, t)=0 \\
T(y, 0)=T_{\infty}, \quad \frac{\partial T}{\partial y}(0, t)=0, \quad T(\infty, t)=T_{\infty} .
\end{array}\right.
$$

In the laser welding simulation, it is essentially the temperature change, translated into thermal expansion in the mechanical analysis, which becomes external load in the model. The temperature is usually linearly ramped to the prescribed value and thereafter it is followed by a constanttemperature stage. This method has been used quite frequently in the $2 \mathrm{D}$ analytical form proposed by Lee et al (2002). The other method, prescribed heat input is the most commonly used 
method. It applies the heat input as a heat flux at integration points, which is then converted to the nodes as temperature loads. The most commonly used heat source of this kind have a Gaussian distribution. Numerical technique is used to set the geometry, the mesh and the boundary of a 3D computational domain. The fluent running allows one to obtain the numerical solution of the flow equations by finite volume discretization. For all the treated cases, the calculation was carried out with a uniform structured mesh composed of hexahedral cells. This grid was selected among others, following the calculations carried out with finer grids composed of 15400 cells and 75450 cells. The differences between the results obtained using these three grids are lower than $5 \%$ for all the flow sizes. The choice of the grid was thus based on the lowest computing time (Kamel et al 2008).

To eliminate the enthalpy in the keyhole boundary layer, we can write the following expression. Here $h=C_{p} T, C=$ const., we get

$$
\frac{\partial T}{\partial t}=\frac{\partial}{\partial y}\left(\alpha \frac{\partial T}{\partial y}\right)+\frac{v}{C_{p}}\left(\frac{\partial u}{\partial y}\right)^{2},
$$

where $\alpha=\frac{k}{C_{p}}$ is the thermal diffusivity and $v=\frac{\eta}{\rho}$ is the kinematic viscosity of the fluid residing in the volume of the keyhole. Due to incompressiblity assumption, the similar solution is valid provided the Eckert number $E$ is small compared to unity. The Eckert number is a dimensionless number used in heat dissipation of calculation associated with fluid flow. It is based on relationship between a flow kinetic energy and enthalpy. It is defined as $E=\frac{V^{2}}{2 \cdot C_{p} \Delta T}$. This is ratio of the kinetic energy and enthalpy. $V=\sqrt{2 \times E \times C_{p} \times \Delta T}$ is characteristic velocity of the liquid metal flow within the keyhole. $C_{p}=\frac{V^{2}}{2 \times E \times \Delta T}$ is the specific heat at constant pressure of the flow. $\Delta T=\frac{V^{2}}{2 \times E \times C_{p}}$ is the characteristic temperature difference in the flow. The turbulent Prandtl number $\left(P r_{t}\right)$ is a non-dimensional term defined as the ratio between the momentum eddy diffusivity and the heat transfer eddy diffusivity. It is useful for solving the heat transfer problems encountered in turbulent boundary layer flows. The simplest model for $\operatorname{Pr}_{t}$ is the Reynolds analogy, which yields a turbulent Prandtl number close to 1. From experimental data obtained by Fabbro et al (2005), $P r_{t}$ has an average value of 0.85 , but it ranges from 0.7 to 0.9 depending on the Prandtl number of the given fluid. The introduction of eddy diffusivity and subsequently the turbulent Prandtl number works as a way to define a simple relationship between the excess shear stress and heat flux present in a turbulent flow. If the momentum and thermal eddy diffusivities are zero (no apparent turbulent shear stress and heat flux) then the turbulent flow equations reduce to laminar type of expressions. We can define the turbulent Prandtl number through the eddy diffusivities and connect it to the momentum and thermal equations.

$$
u \frac{\partial T}{\partial x}+v \frac{\partial T}{\partial y}=\frac{\partial}{\partial y}\left[\left(\alpha+\frac{\delta_{M}}{\operatorname{Pr}_{t}}\right) \frac{\partial T}{\partial y}\right]
$$

where $u$ and $v$ are the turbulent shear stresses in the keyhole, $\delta_{M}$ is the momentum transfer in the fluid. In a special case, where both Prandtl number and turbulent Prandtl number are equal to one, the velocity and the temperature profiles become identical. This simplifies the solution to the heat transfer problem. If the Prandtl and turbulent Prandtl numbers differ from one another than the momentum and eddy diffusivities, solutions are found for momentum and thermal equations. 
Vorticity and entropy production in terms of $E$ are easily evaluated. For the circulation of vorticity, we can write the governing expressions as defined by Hu \& Tsai (2003)

$$
\begin{gathered}
\int_{c} \frac{d u}{d t} \cdot d s=\int_{A} \nabla \times\left(-\frac{1}{\rho} \nabla p+\nabla \Phi\right) \cdot n d S=\int_{A} \frac{1}{\rho^{2}}(\nabla \rho \times \nabla p) \cdot n d S=0, \\
\left\{\begin{array}{c}
\frac{d \vec{\omega}}{d t}=\frac{d \vec{\omega}}{\partial t}+\vec{V} \cdot(\vec{\nabla} \vec{\omega})=(\vec{\omega} \cdot \vec{\nabla}) \vec{V}-\vec{\omega}(\vec{V} \cdot \vec{V}) \\
+\frac{1}{\rho^{2}} \vec{\nabla} \rho \times \vec{\nabla} p+\vec{\nabla}\left(\frac{\vec{\nabla} \cdot \tau}{\rho}\right)+\vec{\nabla} \times \vec{B}
\end{array}\right.
\end{gathered}
$$

Here $\vec{V}$ is the velocity vector, $\nabla p$ is the pressure gradient, $\tau$ is the viscous stress tensor and $\vec{B}$ is the liquid vapour force term, $d S$ is an element along the closed contour, $n$ is normal orientation of closed counter $d S$ and $\Phi$ is the potential for the vapour pressure. The term $\frac{d \vec{\omega}}{d t}=\frac{\partial \vec{\omega}}{\partial t}+\vec{V} \cdot(\vec{\nabla} \cdot \vec{\omega})$ is the material derivative of the vorticity vector $\vec{\omega}$. It describes the rate of change of vorticity of a fluid particle within the keyhole volume (or in other words the angular acceleration of the liquid-vapour phase). This can change due to the unsteadiness in the flow expressed by $\frac{\partial \vec{\omega}}{\partial t}$ (unsteady state term which indicates that the vorticity is not a linear function of time as it can be derived from the conservation of momentum). It can as well be obtained from the motion of a fluid particle include keyhole channel. $\vec{V} \cdot(\vec{V} \cdot \vec{\omega})$ is the convection term for the liquid-vapour phase. The first term on the RHS of the vorticity equation (17) $(\vec{\omega} \cdot \vec{V}) \vec{V}$, describes the stretching or tilting of vorticity due to prevailing velocity gradients. The next term $\vec{\omega}(\vec{V} \cdot \vec{V})$ describes stretching of vorticity due to flow compressibility. The third term $\frac{1}{\rho^{2}} \vec{\nabla} \rho \times \vec{\nabla} p$ is a baroclinic term. It accounts for the change in the vorticity due to intersection of density and temperature surfaces inside the keyhole channel. The term $\vec{\nabla} \times\left(\frac{\vec{\nabla} \cdot \tau}{\rho}\right)$ accounts for the dispersion of vorticity due to viscous effects. $\vec{\nabla} \times \vec{B}$ term deals with changes occurring due to prevailing liquid-vapour forces. The vorticity equation describes the evolution of the vorticity $(\vec{\omega})$ of a fluid element as it revolves around. The vorticity equation can be derived from the conservation of momentum equation. In its general vector form, it may be expressed as follows

$$
\vec{\omega}=\nabla x \cdot \vec{w}=-\frac{\partial u}{\partial y}\left|=\frac{U}{(\pi v \cdot t)^{0.5}} \exp ^{-\eta^{2}}\right|=\left(\frac{C_{p} T_{\infty}}{\pi v \cdot t}\right)^{0.5} \exp ^{-\eta^{2}}
$$

For entropy calculations, we need the temperature gradient in the volume of the keyhole. This can be achieved by executing following mathematical steps:

$$
\begin{gathered}
\nabla T=\frac{\partial T}{\partial y} \mid=-\frac{U^{2} \exp ^{-\eta^{2} e r f} \eta}{C_{p}(\pi v \cdot t)^{0.5}}, \\
\Phi=v\left(\frac{\partial u}{\partial y}\right)^{2}=\frac{\rho U^{2}}{\pi t} \exp ^{-2 \eta^{2}}
\end{gathered}
$$




$$
\rho T=\frac{\rho U^{2}}{\pi t} \exp ^{-2 \eta^{2}}+\frac{k U^{4} \exp ^{-2 \eta^{2}(\operatorname{erfc} \cdot \eta)^{2}}}{C_{p}^{2} \cdot \pi v t(T)}
$$

The expression for Cp can be described by

$$
C_{p}=\frac{E \cdot \exp ^{-2 \eta^{2}}}{\pi\left\{1+\frac{E}{2}\left[1-(\operatorname{erfc} \cdot \eta)^{2}\right]\right\}}\left(1+\frac{E(\operatorname{erfc} \cdot \eta)^{2}}{1+\frac{E}{2}\left[1-(\operatorname{erfc} \cdot \eta)^{2}\right]}\right),
$$

$\operatorname{Pr}=1 \Rightarrow 1-\operatorname{erfc} \cdot \eta \sim \frac{1}{\pi^{0.5} \cdot \eta} \exp ^{-\eta^{2}}$ at, $\eta \rightarrow \infty$. This rapid exponential decay is typical of both steady and unsteady high $R_{e}$ number boundary layers encompassed by a viscous layer.

A transformation is described, which relates the Mach number of the flow to the vorticity. These are expressed in terms of Reynolds number for different sizes of keyhole mouth. For compact flow fields, the apparent sound source is of quadrupole type and varies linearly with the vorticity. Therefore, it changes linearly with the flow velocity. It is found that at low Mach numbers associated with free-space turbulence, the generated velocity of sound is related to the vorticity correlation tensor. It is observed that larger the radius of the keyhole more is the magnitude of vorticity production. The vorticity production increases sharply when the corner angle of keyhole is of higher magnitude. However, at these larger angles the rate of vorticity production increases and ultimately approaches a constant value. Strong shock waves induce faster vorticity. Only exception occurs when the slipstream originating from the shallow corner attaches to the downstream wall of the keyhole. It is found that the vorticity induced by the slipstream contains a large fraction of the total vorticity. Therefore, the slipstream is an important source of vorticity in comparison with baroclinic effects caused by shock wave diffraction.

\section{Results and discussion}

In fluid dynamics, vorticity is well-known as curl of the fluid velocity. It can also be considered as the circulation per unit area at a point in a liquid-vapour velocity field. It is a vector quantity, whose direction lies along the axis of the fluid's rotation. For a two-dimensional flow, the vorticity vector is perpendicular to the plane. The various hydrodynamic transport phenomena calculations are based on dimensionless numbers which is based on the detail mechanism of keyhole generation due to vapour bursts described by Fabbro et al (2004). This is followed by rapid collapse of the keyhole channel due to excess vapour recoil pressure. In general, as shown in figure 2 , the vorticity $(\omega)$ in radian per second is a powerful concept in case of low viscous high Reynold number flows. In such cases, even when the velocity field is relatively complicated, the vorticity field can well be approximated to zero everywhere except in small regions of spatial confines. Related concepts include vortex-line, a line that is everywhere tangential to the local vorticity. Similarly, a vortex-plane in the fluid is formed by all vortex-lines passing through a closed curve in the keyhole. The strength of a vortex is the integral of the vorticity across a cross-section of the keyhole. It is same everywhere along the keyhole (because vorticity has zero divergence). A consequence of Helmholtz theorem (or equivalently Kelvin's circulation theorem) states that in an inviscid fluid, the strength of the vortex line remains constant with time. In figure 2, it is pointed out that the degree of vorticity sharply increases at high Reynolds numbers. It also depends on the radius of the keyhole mouth, which generates the vapour recoil pressure across the keyhole axis. Vorticity of the melt has been calculated as a function of Reynolds number. It shows that vorticity increases with Reynolds number for various Mach numbers. The 
(a)

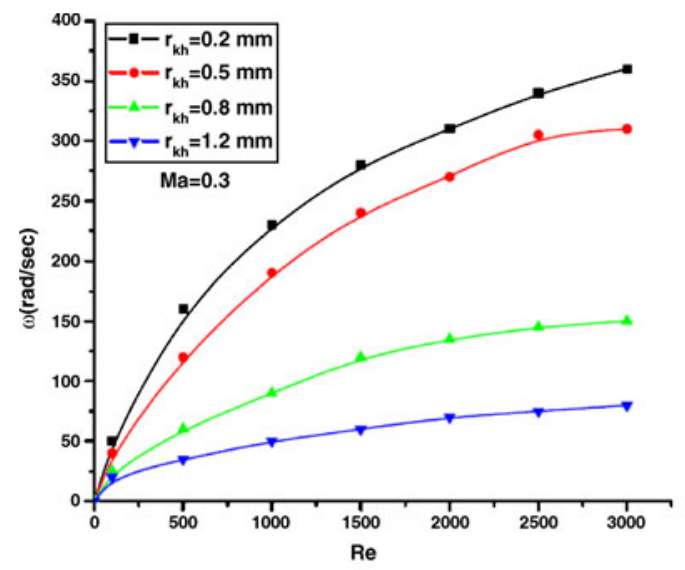

(c)

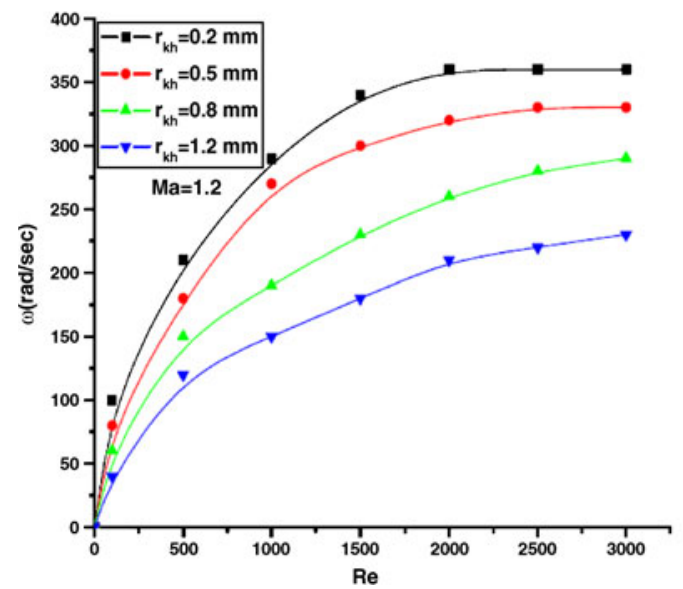

(b)

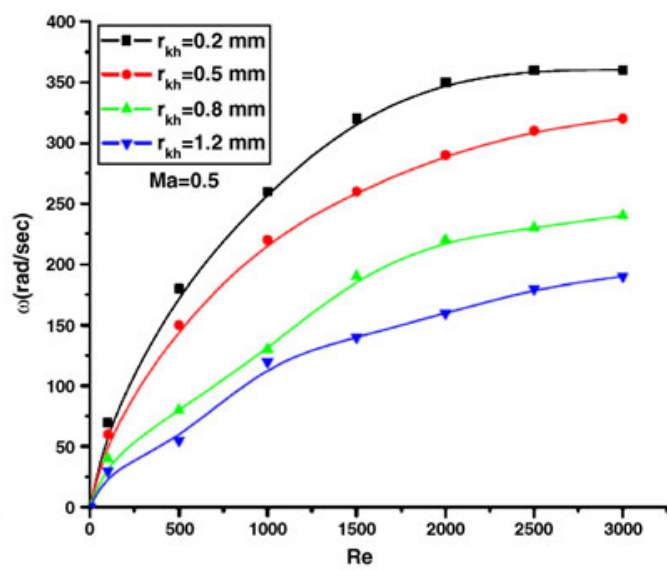

(d)

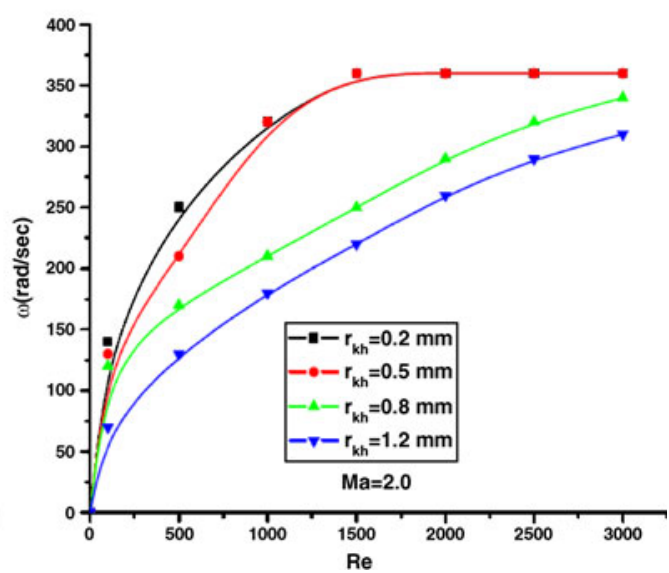

Figure 2. Renoylds number as a function of vorticity for four different values of the keyhole mouth radius at (a) $\mathrm{Ma}=0.3,(\mathbf{b}) \mathrm{Ma}=0.5,(\mathbf{c}) \mathrm{Ma}=1.2$ and (d) $\mathrm{Ma}=2$ for constant laser power $\mathrm{P}=2.5 \mathrm{~kW}$.

power of the laser is kept constant at $2.5 \mathrm{~kW}$. It is a finding that the Mach number does not influence vorticity of the melt significantly a shown in figures $2 \mathrm{a}-\mathrm{d}$. Higher Mach number is a strong hydrodynamic dimensionless quantity which accounts for the enhanced vorticity. It is found that for the higher order of keyhole with a size $r_{k h}=1.2 \mathrm{~mm}$, the average vorticity reduces by 5 times when compared to the vorticity at $r_{k h}=0.2 \mathrm{~mm}$. The vorticity shows ascendancy with increase in Mach number. In all cases in figures $2 \mathrm{a}-\mathrm{d}$ it is found that Mach number is an important parameter for the enhancement of the vorticity as a function of Reynolds number. The magnitude of vorticity slightly increases for higher Mach number due to the generation of strong shock waves in the keyhole. The free-vortex flow of the liquid-vapour in the keyhole channel is derived from the vorticity transport equation. This equation is the curl of the Eulerian momentum equation. A barotropic flow in keyhole channel ensues with vanishing source terms. The vorticity associated with irrotational flow is zero. For a two-dimensional liquid-vapour flow in the keyhole channel, the vorticity acts as a measure of the local rotation of fluid elements. Note that the vorticity 
does not imply anything about the global behaviour of a fluid. It is possible for a fluid traveling in a straight line to posses vorticity. It is also possible for a fluid which moves in a circle to be spatially irrotational. For liquid-vapour substances in conserved force field the expression $\vec{\nabla} \times \vec{B}=0$. A barotropic fluid is defined by the mathematical property $\vec{\nabla} \rho \times \vec{\nabla} p=0$. For an inviscid fluid $\tau=0$. For a constant density fluid, $\nabla \rho=0$, at a given temperature gradient. Vortex dynamics presumes that the vorticity field can be modelled in terms of discrete vortices enclosed within intensive and relevant flows. In general, the presence of viscosity causes a dispersion of vorticity away from these small regions (e.g., discrete vortices) into the general flow field. This can be seen in the keyhole channel from the presence of diffusion term in the vorticity transport equation. Thus, in cases of strong viscous flows, the vorticity will be dispersed through out the flow field. In this case, it will be prudent to consider velocity field rather than look at the vorticity field which becomes less intuitive. In figure 3, the value of Mach number is shown as a function of keyhole size $r_{k h}$ for five different values of $\mathrm{CW} \mathrm{CO}_{2}$ laser power, at a constant external pressure. In the case of keyhole radius ranging between $r_{k h}=0.2-0.4 \mathrm{~mm}$, the Mach number ranges between $1.5 \leq M a \leq 3.2$, implying vapour ejection from the fluid. In the range of keyhole size $0.4 \geq r_{k h} \geq 0.8 \mathrm{~mm}$, the Mach number varies between $0.1 \leq M a \leq 2.7$, indicating subsonic to supersonic transition of vapour flow in the keyhole channel. The value of Mach number linearly decreases for comparatively higher keyhole sizes. This phenomenon explains the hydrodynamic behaviour of liquid-vapour recoil pressure in the keyhole volume. In this case, the keyhole behaves like a nozzle for the recoiling vapour flow. If the keyhole size is small, the fluid flow is enhanced due to high vapour pressure prevailing inside the keyhole channel. This oscillatory hydrodynamic nature of Mach number variation can explain the fluid flow instabilities and collapse of keyhole channel encountered during deep penetration laser beam welding. In figure 4, the results represent keyhole size $r_{k h}$ as a function of Reynolds number for five different values of $\mathrm{CW} \mathrm{CO} 2$ laser power. Turbulent Reynolds number $\operatorname{Re}=\frac{\left[\rho(M a) \times r_{k h}\right] \times \vec{\omega}}{\mu}$ represents the ratio of the inertial force to the viscous force in terms of vorticity $\vec{\omega}$. It reflects the degree of turbulence within the liquid-vapour phase in a keyhole channel. In our case, we

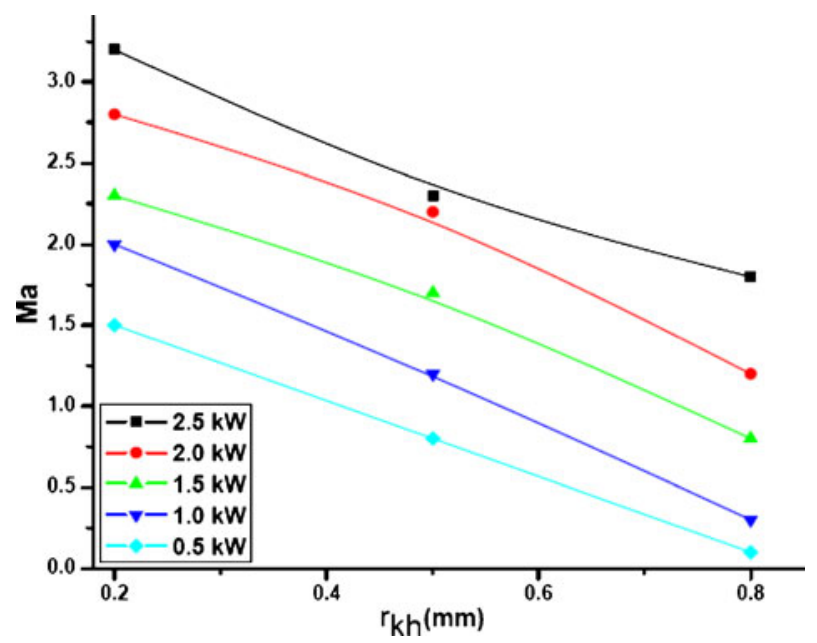

Figure 3. Size of keyhole mouth as a function of Mach number variation for five different values of laser power. 


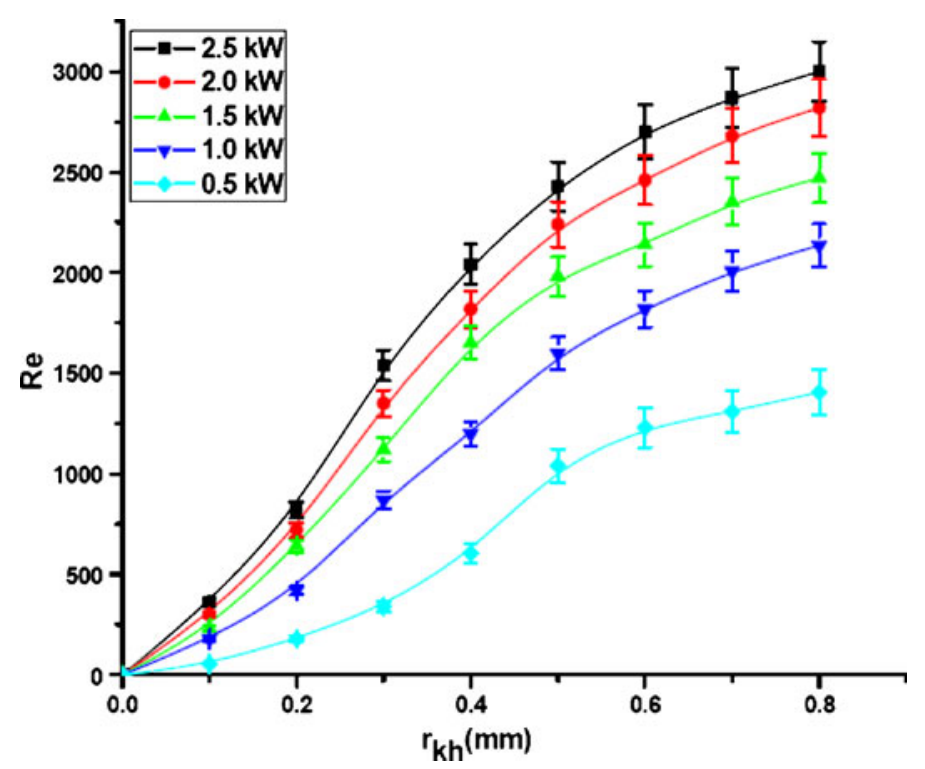

Figure 4. Size of keyhole mouth as a function of Reynold number for five different values of delivered $\mathrm{CW} \mathrm{CO}_{2}$ laser power at constat external pressure.

consider the velocity of the liquid-vapour phase equal to the Mach number. In all the cases, the increase in Reynolds number results in widening of keyhole due to prevalence of relatively higher turbulence. The incident laser power is also an important parameter that enhances the turbulent motion in the keyhole. It is also directly related to the rate of melting of the material. Results shown in figure 5 represent keyhole depth as a function of Rayleigh and Mach numbers for a laser power of $1 \mathrm{~kW}$ and welding speed $12 \mathrm{~m} / \mathrm{min}$. The Rayleigh number shows conductive mode of heat transfer inside the keyhole depth $0 \geq h_{k h} \geq 0.3 \mathrm{~mm}$. In the Rayleigh number range $5 \times 10^{7} \geq R a \geq 2 \times 10^{7}$, the mode of turbulent convective heat transfer dominates at the keyhole depth $0.3 \geq h_{k h} \geq 0.5 \mathrm{~mm}$. In the bottom of the keyhole $h_{k h}=0.5-0.8$ the Raleigh number again reduces to $\mathrm{Ra}=10^{7}$ and the process reverts from convective to conductive heat transfer mode. The Mach number falls rapidly with increasing depth of the keyhole channel due to interference of the flow pattern. Inside the keyhole depth, the Mach flow pattern can be divided into three distinct zones i.e., subsonic, sonic and supersonic flow. While traversing down the keyhole, the Mach flow pattern, which is supersonic in keyhole mouth, gradually becomes sonic at intermediate levels and finally turns into subsonic in bottom most part of the keyhole. The knowledge of these hydrodynamic parameters is essential to optimize the weld quality in laser beam welding. If the Mach number of liquid-vapour phase exceeds a certain value, it can cause formation of pores in the weld seams and ejection of melt from melting bath. This effect can reduce the mechanical resistance of welding quality.

Marangoni convection in the weld pool can affect its depth significantly, which is often critical in welding. Marangoni convection in the weld pool is induced by surface-tension gradients along the pool surface, which drives the liquid flow at the pool surface from low surface tension to high. The surface-tension gradients are induced by the temperature gradients along the pool surface. Laser-induced heating makes the temperature of the pool surface significantly higher at the center of the keyhole than at the edge of the keyhole wall. In the configuration of a weld pool, 


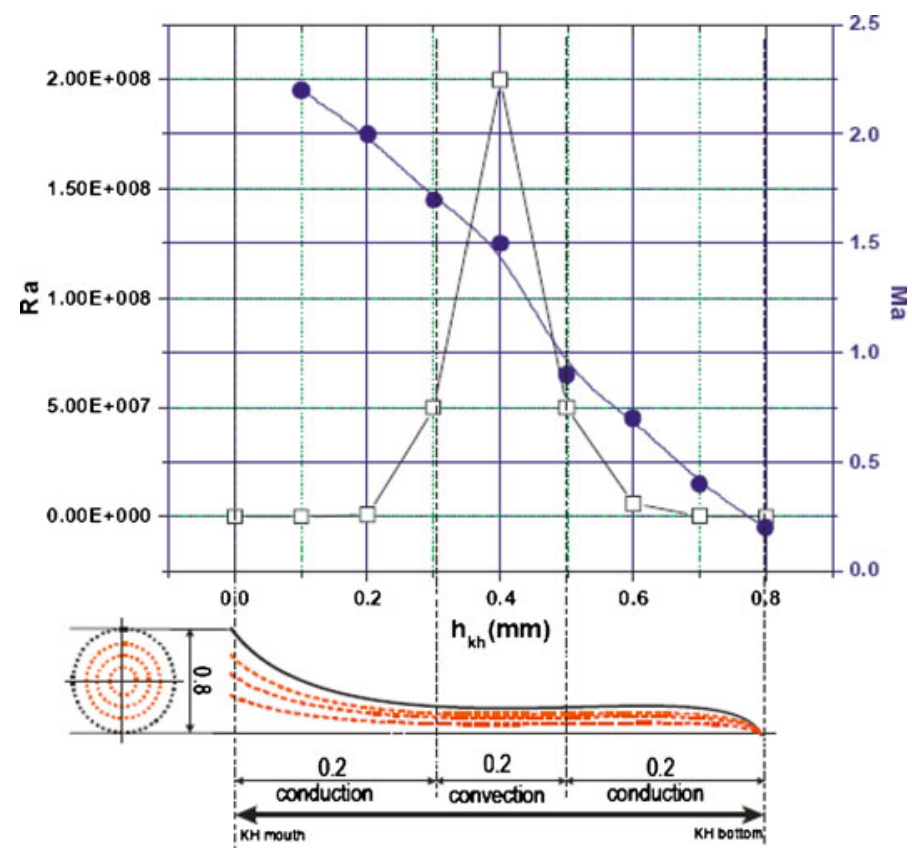

Figure 5. The profile of Raleigh and Mach number variation as a function of keyhole depth.

buoyancy convection occurs in the same direction as Marangoni convection. Therefore, it should be checked whether the convection observed is driven primarily by Marangoni convection or buoyancy convection. The dynamic Bond number is often used as an indication of the relative strength of buoyancy convection to Marangoni convection. It is defined as $B o-\beta \rho g \times L_{2} /(-\partial \gamma / \partial T)$, where $\beta$ is the thermal expansion coefficient of the melt, $\rho$ the density of the melt, $g$ the gravitational acceleration, $L$ the characteristic length of the pool, and $\partial \gamma / \partial T$ is the temperature coefficient of surface tension of the melt. As shown in the figure 4, the region of the melt of the flow loops are very close to the pool surface, where fluid flow is much faster and the flow lines are much more closely spaced than in the bulk pool. This suggests that convection in these pools is dominated by Marangoni convection. When the temperature difference between the center of the pool surface and the edge is high, the flow loops are stable and axi-symmetric. When the temperature difference is reduced beyond a certain point, however, convection weakens and the flow loops begin to lose stability and axi-symmetry. Therefore, if the buoyancy force is significant then the Marangoni convection appears in the centre of the keyhole and it disappears away from the keyhole region.

\subsection{Validation of the model}

Finally, the simulation results are compared with the experimental data. The effectiveness of the developed computational procedures have been confirmed. The contour for the boiling point of the material is defined as the keyhole boundary where the region between the boiling and melting indicate formation of weld pool. Moreover, when the Rayleigh number, is below a critical value, the heat transfer occurs primarily in the form of conduction. When it exceeds this critical value, heat transfer becomes convection dominated. This weld pool has the shape very similar 
to the weld pool shape presented by Sudink et al in which the contribution of transport such as conductivity, fluid flow and convexity in the weld pool shape were taken into account to define the fluid flow in weld pool (Dowden 2002). It is reasonable to analyse the figures $1 \mathrm{a}-\mathrm{d}$ we are able to estimate another weld pool cross section with a different laser power. To validate this assumption, a laser weld cross section in a $5 \mathrm{~mm}$ plate was chosen. For extending this to a 3-D modelling, one could use the data of the weld cross section from an experiment for defining the absorption coefficient for each element along the thickness. So an absorption profile is easily obtained as a function of penetration depth, which is helpful for prediction of weld cross sections. The Reynolds and Mach numbers decrease with radius of keyhole for various laser powers. Calculated results of the Reynolds number in the keyhole and weld pool are compared with the results presented by $\mathrm{Hu} \&$ Tsai (2003). Heat transfer from the laser keyhole in the weld pool and details of the fluid flow play an important role in determining weld shape and size. Experimental and the finite volume methods are utilized to investigate the thermal phenomena during continuous laser keyhole welding. It has been found that the shape and size of the molten pool in the work piece are affected by welding parameters such as welding speed and the incident laser power as studied by Kaplan et al (2002). It has been observed that the temperature coefficient of surface tension and Marangoni convection are sensitive parameters that describe the fluid flow in the weld pool region. These coefficients also affect the pattern of the fluid flow in the molten pool.

\section{Conclusions}

The strength of vorticity across a cross-section of the keyhole channel has been described in terms of angular velocity of the melt. It is equal everywhere in the keyhole as vorticity has zero divergence. The magnitude of vorticity gets enhanced with higher Reynolds number. It also depends on the radius of the keyhole mouth which experiences vapour recoil pressure along the keyhole axis. The resulting increase in the curvature of the fluid momentum is related to the rapid temporal collapse of the keyhole. Present study also addresses vorticity dynamics in liquid-vapour flow occurring in the keyhole channel. The Mach number is considered as a strong hydrodynamic indicator connected to enhanced vorticity in the keyhole channel. It also explains the rapid oscillatory motion in the liquid-vapour phase. The expression of thermal diffusivity and turbulent Prandtl number defines relationship between the excess shear stress and heat flux in the keyhole region. In the analytical calculations, the Mach number is found to be linearly decreased with increasing size of keyhole mouth. The hydrodynamic treatment of liquid-vapour recoil pressure acting along the cross section of keyhole charecterizes nozzle action associated with vapour recoil flow. It is assumed that the velocity of the liquid-vapour phase is equal to the supersonic flow present in the keyhole volume. The Reynolds number increases for larger keyhole sizes due to higher turbulence. The laser power is also an important parameter which influences the variation in turbulence motion in the liquid-vapour phase in keyhole region. This is again directly proportional to the rate of melting and evaporation of the material. In addition, surface tension forces acting with shear force caused by intense turbulent vorticity brings out rapid collapse of the keyhole walls. This phenomenon is more prominent at higher Mach numbers. In the experiments, the time for the complete collapse of keyhole after the appearance of protrusion was about $0.2 \mu \mathrm{s}$. This number showed reasonable correlation between the experiment and our computations in which vorticity and dimension of keyhole mouth are functions of Reynolds number and Mach number, respectively. 
The authors would like to thank Prof. A V Fedin and Dr. A A Govrilov from Kovrov State Technological Academy, Russia and VS Golubev from Institute on Laser and Infromation Technologies, Russian Academy of Science (RAS), Russia for useful discussions.

\section{References}

Dowden J 2002 Interaction of the keyhole and weld pool in laser keyhole welding. J. Laser Applications 14(4): 204-209

Fabbro R, Hamadou M, Coste F 2004 Metallic vapor ejection effect on melt pool dynamics in deep penetration laser welding. J. Laser Applications 16(1): 16-19

Fabbro R, Slimani S, Coste F, Briand F 2005 Study of keyhole behaviour for full penetration Nd-Yag CW laser welding. J. Phys. D: Appl. Phys. 38:1881-87

He X, Fuerscbach P W, DebRoy T 2003 Heat transfer and fluid flow during laser spot welding. J. Phys. D: Appl. Phys. 36:1388-94

Hu J, Tsai H L 2003 Modeling the transport phenomena during dual beam laser welding process. Lasers and Electro-Optics Europe 22: 562-566

Kamel A, Wacef B S, Hatem M, Georges L, Michel A 2008 Modelling of $\mathrm{CO}_{2}$ laser welding of magnesium alloys. Opt. Laser Technol. 40: 581-88

Kaplan A A 1994 Model of deep penetration laser welding based on calculation of the keyhole profile. $J$. Phys. D: Appl. Phys. 27: 1805-14

Kaplan A F H, Masami M, Seiji K, Matsunawa A 2002 Unbounded keyhole collapse and bubble formation during pulsed laser interaction with liquid zinc. J. Phys. D: Appl. Phys. 35: 1218-1228

Klein T, Vicanek M 1996 Forced oscillations of the keyhole in penetration laser beam welding. J. Phys. D: Appl. Phys. 29: 322-332

Lee J Y K, Sung O H, Farson D F, Yoo C D 2002 Mechanism of keyhole formation and stability in stationary laser welding. J. Phys. D: Appl. Phys. 35: 1570-1576

Matsunawa A 2001 Problems and solutions in deep penetration laser welding. Sci. Technol. Welding and Joining 6(6): 351-354

Mi B, Charles U I 2004 Three-dimensional ray tracing of laser ultrasound for weld penetration sensing. $J$. Acoust. Soc. Am. 115: 1565-1571

Seto N, Seiji K, Matsunawa A 2000 High-speed simultaneous observation of plasma and keyhole behaviour during high power $\mathrm{CO}_{2}$ laser welding: Effect of shielding gas on porosity formation. J. Laser Applications 12(6): 245-250

Zhou J, Tsai Hai-Lung, Wang Pei-Chung 2006 Transport phenomena and keyhole dynamics during pulsed laser welding. J. Heat Transfer 128(7): 680-690 